\title{
MiR-365a-3p suppresses proliferation and invasion of Hep-2 cells through targeting ten-eleven translocation 1 (TET1)
}

\author{
J. LI ${ }^{1, *}$, N. SHEN ${ }^{2,3, *}$, G. P. BAI ${ }^{1}$, X. S. HUANG ${ }^{2, *}$ \\ ${ }^{1}$ Department of Otolaryngology, Qingpu Branch of Zhongshan Hospital, Fudan University, Shanghai, China; ${ }^{2}$ Department of Otolaryngol- \\ ogy, Zhongshan Hospital, Fudan University, Shanghai, China; ${ }^{3}$ State Key Laboratory of Molecular Engineering of Polymers, Fudan University, \\ Shanghai, China
}

*Correspondence: huang.xinsheng@zs-hospital.sh.cn

${ }^{*}$ Contributed equally to this work.

Received November 19, 2017 / Accepted February 28, 2018

\begin{abstract}
miRNAs are among the most important factors that regulate gene expression. Bioinformatic analysis supports our prediction that miR-365a-3p affects tumor biological processes through TET1, so TET1 interference and miR-365a-3p inhibitor constructs were generated. qRT-PCR verified the expression level of miR-365a-3p and TET1 in Hep-2 and BESB-2B cells and qRT-PCR and Western blot were used to confirm the TET1 expression level in Hep-2 and miR-365a-3p inhibitor cells. Cell proliferation, cell cycle progression and cell invasion were further studied to identify the relationship between TET1 and miR-365a-3p. Luciferase reporter gene assays were used to find the binding site of miR-365a-3p in the 3'-UTR (3'-untranslated region) of the TET1 mRNA. TET1 was weakly expressed in Hep-2 cells and highly expressed in BESB-2B cells, while miR-143-3p and miR-365a-3p were highly expressed in Hep-2 cells and lowly expressed in BESB-2B cells. Inhibiting miR-365a-3p could up-regulate the expression of TET1 and the negative effects of miR-365a-3p on cell proliferation, cell cycle progression and cell invasion could be abolished by TET1 interference. The miR-365a-3p binding site is in the 3'-UTR of the TET1 mRNA. TET1 is one of miR-365a-3p's targets and miR-365a-3p regulates the biological behavior of laryngeal cancer by down-regulating TET1.
\end{abstract}

Key words: TET1, miR-365a-3p, laryngeal cancer

Despite the development of surgical and chemo-radiotherapy, the morbidity of laryngeal cancer has increased in recent years [1]. Recurrence and remote metastasis after treatment play important roles in the poor prognosis of laryngeal cancer $[2,3]$. The effects of oncogenes and tumor suppressor genes on this cancer have been widely studied, but the mechanism involved in carcinogenesis is unclear. The mechanism underlying laryngeal cancer proliferation and metastasis should therefore be studied.

MicroRNAs (miRNAs) are small, single stranded, non-coding RNAs that are important gene regulatory factors ranging from 18-24 nucleotides in length [4, 5]. Accumulating evidence has shown that miRNAs play an important role in regulating biological processes and modulating the cell cycle, phenotype, cell invasion and differentiation [6, 7]. It was reported that aberrant expression of miRNAs was related to tumor growth and development [8], and it was also shown to have an impact on laryngeal cancer $[9,10]$.
TET1 is a member of the ten eleven translocation (TET) family that provided a mechanistic basis for the long-hypothesized active DNA demethylation pathway. TET1 was found to be absent or weakly expressed in many cancers $[11,12]$. It can be defined as a tumor suppressor. In the absence of TET1, tumor cells have the ability to invade and metastasize $[13,14]$ because of the hypermethylation status of its promoter [15], and because miRNAs target the 3'UTR of TET1 and inhibit TET1protein expression [14]. Herein, we found miR-365a-3p directly targeted TET1 and that downregulation of miR-365a-3p increased TET1 expression and decreased proliferation and invasion of Hep-2 cells in vitro.

\section{Material and methods}

Cell lines and cell culture. The human bronchial epithelium (BESB-2B) and Hep-2 laryngeal squamous carcinoma cell line used in this study were purchased from BioVector 
NTCC Inc. (Beijing, China) and maintained in Dulbecco's modified Eagle's medium (DMEM) supplemented with 10\% fetal bovine serum (FBS) at $37^{\circ} \mathrm{C}$ in a $5 \% \mathrm{CO}_{2}$ incubator; $1 \%$ penicillin-streptomycin was used to inhibit bacterial growth.

Construction of interference vectors and lentivirus packaging for TET1 gene. Three TET1 shRNAs were designed based on TET1(NM_030625.2). These were connected to the pds019-pl6.3-SHRNA-BSD vector after annealing. The sequencing results verified the identity of CL946-1, CL946-2 and CL946-3 constructs which were transfected into Hep-2 cells via plasmids. qPCR was used to detect interference efficiency. Results showed that the interference efficiency of sh1 was greater than $70 \%$, and primers used in this study are listed in Table 1.

TET1 related miRNA screening. The miRNAs associated with TET1 were predicted by TargetScan. We selected the species as "human" and entered the human gene symbol as "TET1". We predicted all the miRNAs that could associate with the TET1 gene 3'-UTR. siRNA mimics were synthetized, and transfected into Hep-2 cells. Quantitative realtime polymerase chain reaction (qRT-PCR) was used to examine the result. Total RNA was extracted from cultured cells using TRIzol Reagent (Invitrogen, Carlsbad, CA, USA); $12 \mu \mathrm{L}$ of total RNA was used for first-strand DNA synthesis. qRT-PCR analysis then tested the expression levels of miRNAs and TET1 in Hep-2 and BESB-2B cells using the SYBR Green PCR method (Thermo, Waltham,MA, USA). RT-PCR analysis detected the expression of TET1 mRNA in cells treated with anti-miR-143-3p and anti-miR-365a-3p. The TET1 expression level was normalized to beta-actin, and miRNAs were normalized to that of U6. Western blotting analysis then further examined the expression of TET1 in the cells treated with anti-miR-143-3p and anti-miR-365a-3p.

Construction of luciferase vector. As stated in the above section, the binding site for miR-365a-3p in TET1 was predicted to be "GGCATTA". The 3'-UTR sequence was found in NCBI and the binding site was shown to be "GGCATTA" (MUT was "CCGTAAT"). The region from $150 \mathrm{bp}$ upstream to $150 \mathrm{bp}$ downstream in this $300 \mathrm{bp}$ sequence was amplified and cloned in psiCHECK-2 vector. This provided the psiCHECK-2-TET1-UTR-WT and psiCHECK-2-TET1UTR-MUT plasmids.

Cell transfection. Hep- 2 cells $\left(5 \times 10^{5}\right)$ were inoculated in each well of 96-well plates, and transfection was then conducted by Lipofectamine 2000 (Invitrogen,Carlsbad, CA, USA) This was mixed with anti-miR-143-3p and anti-miR$365 a-3 p$ (synthesized by NOVOBIO, Shanghai, China) and the cells were assayed after 24 hours.

Luciferase reporter gene assays. Cells were inoculated in 6-well plates $\left(5 \times 10^{5}\right)$ and Lipofectamine 2000 (Invitrogen, Carlsbad, CA, USA) performed transfection after 24 hours. Cells were divided into four groups: TET1-3'-UTR-WT, TET1-3'-UTR-WT-miR-365a-3p inhibitor, TET1-3'-UTRMUT and TET1-3'-UTR-MUT-miR-365a-3p inhibitor. Standard dual luciferase reporter assay was performed 36 hours post-transfection and all experiments were performed at least three times.

Cell proliferation analysis. Cells were plated on 96-well plates at $5 \times 10^{3}$ cells/well density and $10 \mu$ l Cell Counting Kit-8 (CCK-8 1:10, SAB) was added to each well at 0, 24, 48 and 72 hours after transfection. The cells were then incubated for 1 hour at $37^{\circ} \mathrm{C}$ in a $5 \% \mathrm{CO}_{2}$ incubator and the micro-plate reader measured absorbance at $450 \mathrm{~nm}$.

Cell cycle analysis. Cells were removed from culture plates using trypsinization, washed with PBS and fixed with $100 \%$ ice cold ethanol for 24 hours at $4{ }^{\circ} \mathrm{C}$. After overnight fixation and washing with $\mathrm{PBS}$, the cells were treated with RNase A ( $1 \mathrm{mg} / \mathrm{mL}$, Solarbio) at $37^{\circ} \mathrm{C}$ for 30 minutes and stained with propidiumiodide (PI) $(50 \mathrm{mg} / \mathrm{L}$, 7SeaBiotech) at $4{ }^{\circ} \mathrm{C}$ for 10 minutes in the dark. Each sample was analyzed by flow cytometry (Accuri C6, BD) and PI with red fluorescence was excited by a 488 -nm argon ion laser. The cell cycle distribution was analyzed by FLOWJO software.

Cell invasion analysis. Transwell cells were placed into serum free DMEM and balanced for 30 minutes at $37^{\circ} \mathrm{C}$. The cell density was adjusted to $1 \times 10^{5} / \mathrm{ml}$ and the lower chamber filled with $800 \mu \mathrm{l}$ serum and the upper chamber filled with $200 \mu \mathrm{l}$ serum. The transwell was removed after 24 hour culture. The membrane close to the upper layer of the inner cell was wiped, and the lower layer cells were fixed with $4 \%$ paraformaldehyde for 30 minutes. The cells were then stained with $1 \%$ crystal violet for 10 minutes washed 3 times and examined under the microscope.

Statistical analysis. Each experiment was repeated at least three times and data was analyzed by GraphPad Prism 5 software. T-test and one-way ANOVA compared mean values $( \pm$ SEM, standard error of the mean) and $\mathrm{p}<0.05$ indicated statistically significant differences.

Table 1. TET1 gene primers.

\begin{tabular}{ll}
\hline Primer & Sequence $\left(\mathbf{5}^{\prime} \rightarrow \mathbf{3}^{\prime}\right)$ \\
\hline Sh1-TET1-F & CACCGCAGCTAATGAAGGTCCAGAACGAATTCTGGACCTTCATTAGCTGC \\
Sh1-TET1-R & AAAAGCAGCTAATGAAGGTCCAGAATTCGTTCTGGACCTTCATTAGCTGC \\
Sh2-TET1-F & CACCGCCCAGAAGATTTAGAATTGATCGAAATCAATTCTAAATCTTCTGG \\
Sh2-TET1-R & AAAACCCAGAAGATTTAGAATTGATTTCGATCAATTCTAAATCTTCTGGC \\
Sh3-TET1-F & CACCGCCTCCAGTCTTAATAAGGTTACGAATAACCTTATTAAGACTGGAG \\
Sh3-TET1-R & AAAACCTCCAGTCTTAATAAGGTTATTCGTAACCTTATTAAGACTGGAGC \\
TET1-F & AGTGGTGACTATGCCAGTGC \\
TET1-R & CAGACCCCACATCGCTTTCT \\
\hline
\end{tabular}




\section{Results}

TET1 related miRNA screening. According to bioinformatic analysis, several miRNAs were predicted to associate with TET1. qRT-PCR showed that TET1 was weakly expressed in Hep-2 cells and highly expressed in BESB-2B cells, and miR-143-3p and miR-365a-3p were highly expressed in Hep- 2 cells and weakly expressed in BESB-2B cells (Figure 1A). There was no significant difference in the expression of other candidate miRNAs in these two cell lines. This most likely indicates that miR-143-3p and miR-365a-3p negatively control TET1 expression. qRT-PCR demonstrated that inhibiting miR-365a-3p can up-regulate the expression of TET1, but a miR-143-3p-inhibitor cannot do so (Figure 1B), and this was supported by Western blotting (Figure 1C). In addition, the proliferation rate of Hep- 2 cells decreased after miR-365a-3p inhibitor transfection, but miR-143-3p inhibitor did not affect Hep-2 cell proliferation (Figure 1D). Therefore, miR-143-3p was excluded from further study.
miR-365a-3p down-regulation suppresses proliferation, invasion and affects the Hep- 2 cell cycle through TET1. Down-regulation of miR-365a-3p decreased the proliferation of Hep-2 cells, and we therefore examined whether miR-365a-3p regulated proliferation through TET1. The repression of proliferation caused by miR-365a-3p inhibitor transfection could be counteracted by interfering with TET1 expression $(\mathrm{p}<0.0001$, Figure 2A). Downregulation of $\mathrm{miR}-365 \mathrm{a}-3 \mathrm{p}$ resulted in a smaller proportion of cells in the $\mathrm{G} 2+\mathrm{S}$ cell cycle phases. It also increased the length of the G1 phase. TET1 interference abolished this effect and enhanced the proportion of cells in G2+S (Figure $2 \mathrm{~B}-\mathrm{D})$. This indicated that miR-365a-3p may regulate cell proliferation through TET1 by modulating the cell cycle. Down-regulation of miR-365a-3p inhibited the invasion of Hep-2 cells, but shTET1 transfection abolished this effect (Figure 3 ). We therefore concluded that miR-365a-3p could regulate biological behavior of laryngeal cancer by targeting TET1.

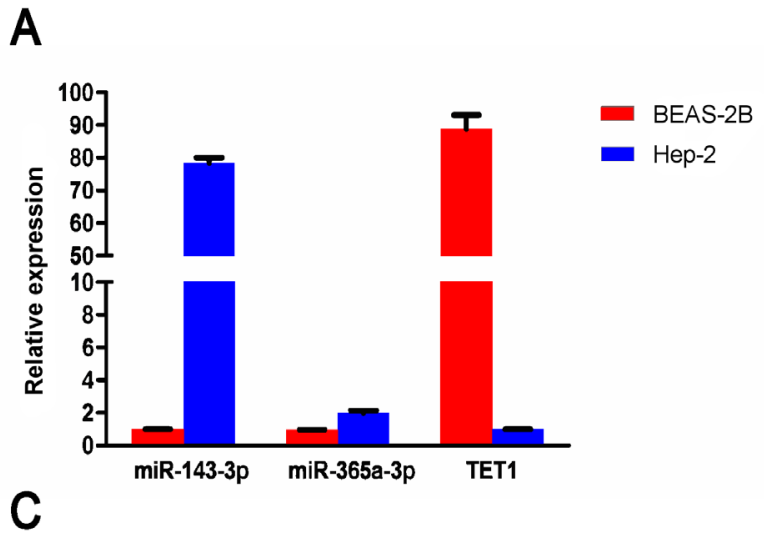

B
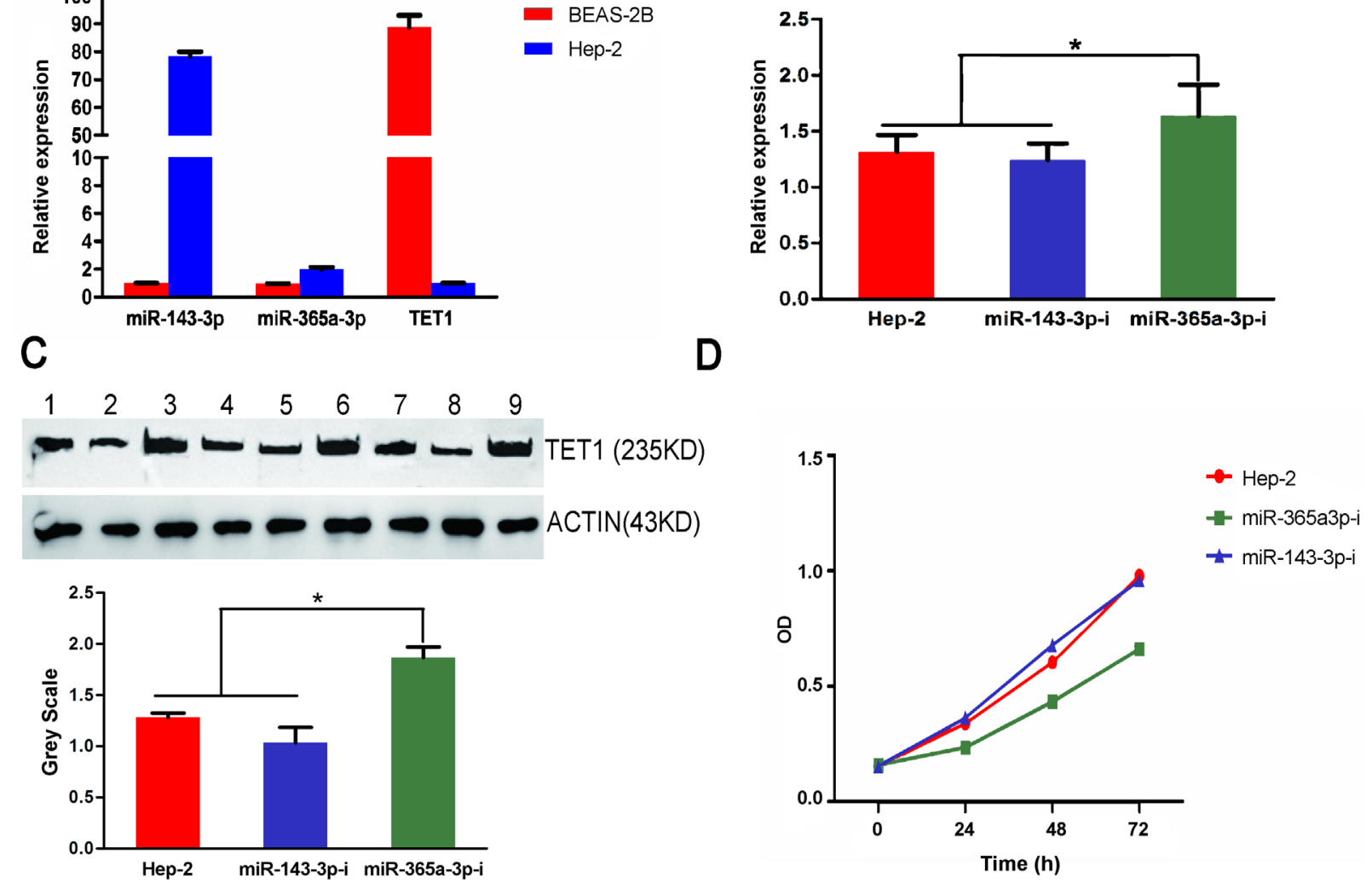

Figure 1. TET1 related miRNA screening. A) qRT-PCR showed that TET1 $\left({ }^{*} \mathrm{p}=0.0023\right)$ was weakly expressed in Hep- 2 cells and highly expressed in BESB-2B cells, and miR-143-3p $\left({ }^{*} p=0.004\right)$ and miR-365a-3p $\left({ }^{*} p=0.0175\right)$ were highly expressed in Hep-2 cells and weakly expressed in BESB-2B cells. B) qRT-PCR demonstrated that TET1 expression could be up-regulated after inhibiting miR-365a-3p $\left({ }^{\star} \mathrm{P}<0.05\right)$, but treatment with miR-143-3p inhibitor did not show the same effect $\left({ }^{*} \mathrm{p}=\mathbf{0 . 7 4 0 4 )}\right.$. C) Western blot showed that TET1 expression could be up-regulated after inhibiting miR-365a$3 p\left({ }^{*} p=0.0237\right)$, but miR-143-3p inhibitor did not have the same effect $\left({ }^{*} p=0.1\right)$. (Hep-2: Land 1,4,7; miR-143-3p inhibitor: Land 2,5,8; miR-365a-3p inhibitor: Land 3,6,9). D) The proliferation rate of Hep-2 cells was decreased after mir-365a-3p inhibitor transfection, but miR-143-3p inhibitor did not have the same effect. 
A

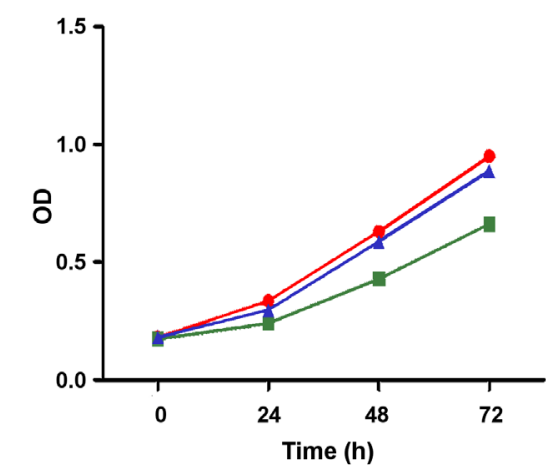

C

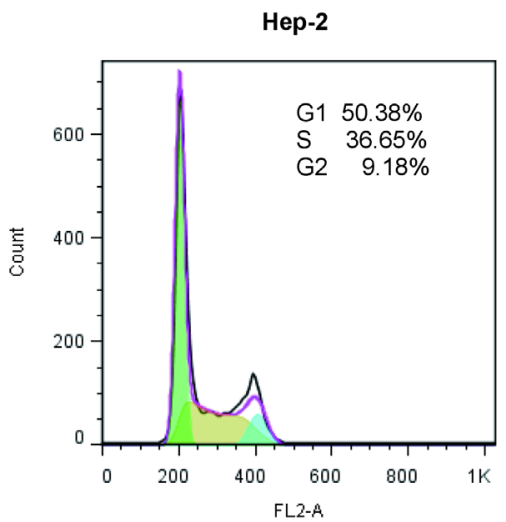

B

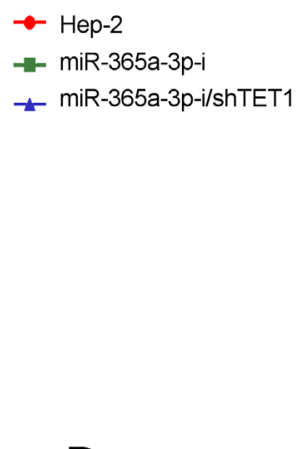

D

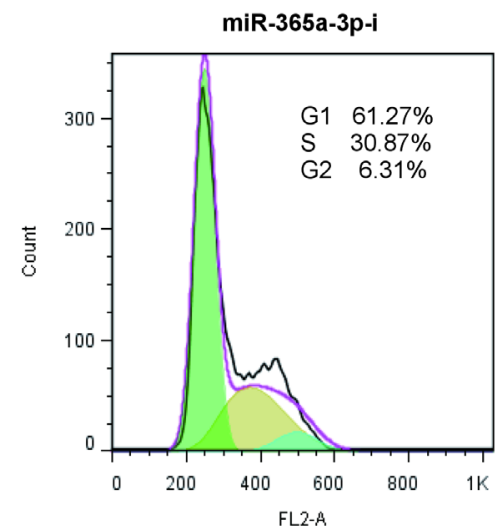

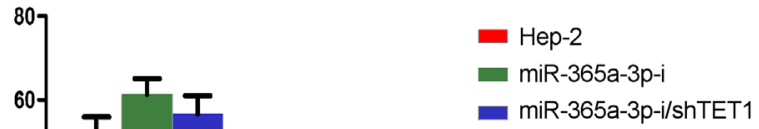

Figure 2. Cell cycle and proliferation analysis. A) The repression of proliferation caused by miR-365a-3p inhibitor transfection could be counteracted by interfering with TET1 expression $(\mathrm{p}<\mathbf{0 . 0 0 0 1})$. B) Down-regulation of mir-365a-3p resulted in a smaller proportion of cells in the $\mathrm{G} 2+\mathrm{S}$ phases of the cell cycle. The number of cells in G1 phase was also increased. TET1 interference could abolish this effect and enhance the proportion of G2+S. (C-E) give the results of flow cytometry for cell cycle analysis. The proportion of each phase was shown.

The binding sites of mir-365a-3p on TET1. We identified the miR-365a-3p binding site in the 3'-UTR of TET1 mRNA. The binding site was between $1343-1349 \mathrm{bp}$ with "GGCATTA" sequence (Figure 4A). The luciferase reporter gene assays demonstrated that the miR-365a-3p inhibitor significantly suppressed the firefly luciferase activities in TET1-3'-UTR-WT and increased the relative value between firefly and renilla luciferase (Figure 4B).

\section{Discussion}

A variety of factors and mechanisms affect the regional growth and metastasis of laryngeal carcinoma [16-18] and miRNAs are among the most important factors regulating gene expression and affecting tumor biological processes $[5,19]$. miRNAs degrade mRNAs or inhibit mRNA translation by binding to the 3'-UTR of target mRNAs [4], and identification of miRNAs and their related genes can lead to new targets for cancer therapy. TET1 is a member of the ten eleven translocation (TET) family which modulates DNA methylation status and regulates gene expression and as a tumor suppressor its expression is reduced in many types of cancer $[11,12,20]$. Moreover, recent studies have confirmed that miRNAs can target the 3'UTR of TET1 and inhibit TET1 protein expression [14, 21, 22].

Herein, miR-143-3p and miR-365a-3p were predicted by bioinformatic analysis to bind to the 3'-UTR of the TET1 mRNA, so we wondered if TET1 could be the target of these two miRNAs. qRT-PCR showed that TET1 was weakly expressed in Hep-2 cells and highly expressed in BESB-2B cells, and miR-143-3p and miR-365a-3p were highly expressed in Hep-2 cells and weakly expressed in BESB-2B cells. Further, qRT-PCR and Western blot showed that miR-365a-3p- inhibitor could up-regulate the expression of TET1, but miR-143-3p-inhibitor did not have the same effect. Moreover, the proliferation rate of Hep-2 cells decreased after miR-365a-3p inhibitor transfection, but miR-143-3p-inhibitor did not have the same effect. Therefore, miR-365a-3p could have a close relationship with TET1; but miR-143-3p was excluded from further study.

Cell proliferation, cell cycle and invasion analysis and TET1 interference were used to investigate whether 

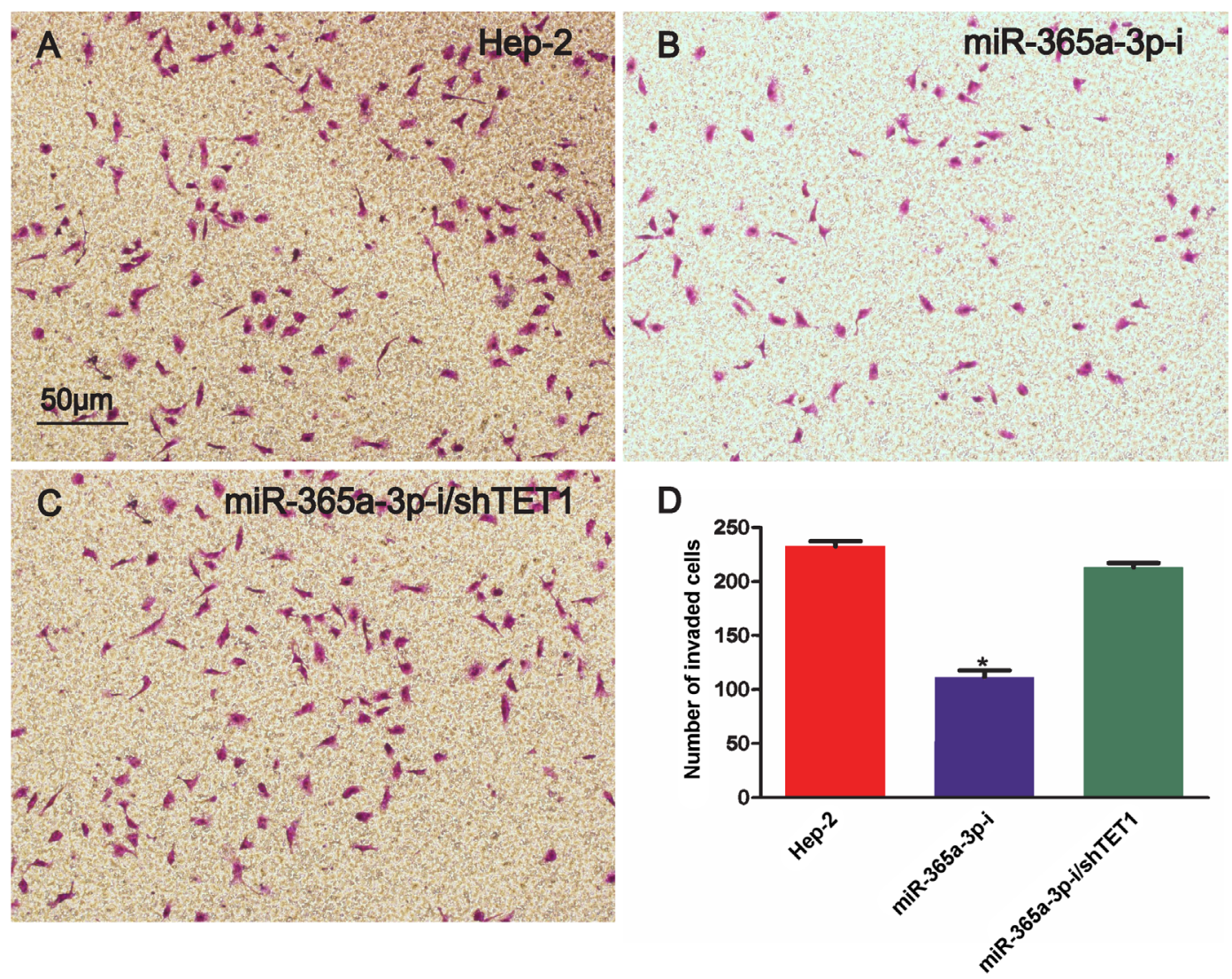

Figure 3. Cell invasion analysis: (A-D) Down-regulation of mir-365a-3p inhibited the invasion of Hep-2 cells, but shTET1 transfection could increase the ability of cell invasion $\left({ }^{*} \mathrm{p}=\mathbf{0 . 0 2 7 3}\right)$.

miR-365a-3p affects the tumor biological processes through TET1. The repression of proliferation caused by miR-365a-3p inhibitor transfection can be counteracted by interfering with TET1 expression. While down-regulation of miR-365a-3p resulted in a smaller proportion of cells in the $\mathrm{G} 2+\mathrm{S}$ phases of the cell cycle, TET1 interference abolished this effect. In addition, the down-regulation of mir-365a-3p inhibited the invasion of Hep-2 cells, but shTET1 transfection increased cell invasive ability.

We also identified the miR-365a-3p binding site in the 3'-UTR of the TET1 mRNA. This was established between 1343-1349 bp with a "GGCATTA" sequence. Therefore, we concluded that miR-365a-3p could regulate the biological behavior of laryngeal cancer by down-regulating TET1. However, the precise TET1 function remains controversial because some studies have reported higher expression of TET1 in some kinds of tumors $[23,24]$. Thus, some mechanisms of the interaction between miRNAs and TET1 remain unknown, and further studies are therefore required.

Finally, and most importantly, our study has established that TET1 is a miR-365a-3p target, and that miR-365a-3p regulates the biological behavior of laryngeal cancer by down-regulating TET1.

\section{A}

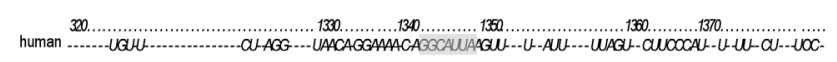

B

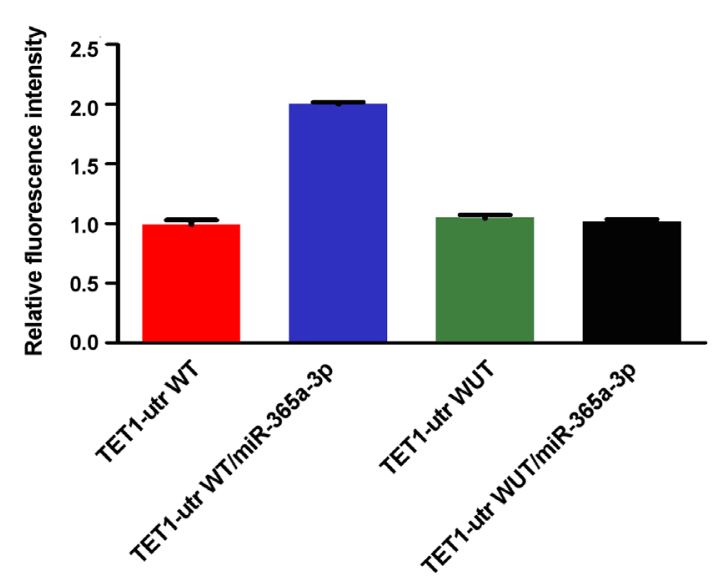

Figure 4. The binding sites of miR-365a-3p on TET1. A) The binding site is between 1343-1349 bp with the sequence 'GGCATTA' B). The luciferase reporter gene assays demonstrated that the miR-365a-3p inhibitor significantly suppressed $\left({ }^{*} \mathbf{p}<0.0001\right)$ the firefly luciferase activity of TET1-3'-UTR-WT and caused the relative value between firefly and renilla luciferase to increase. 
Acknowledgments: This work was supported by the Discipline Leader Training Program of Health and Family Planning Commission, Qingpu District, Shanghai, PR China (WD2015-04) and Shanghai Pujiang Program (18PJD004).

\section{References}

[1] MIRISOLA V, MORA R, ESPOSITO AI, GUASTINI L, TABACCHIERA F et al. A prognostic multigene classifier for squamous cell carcinomas of the larynx. Cancer Lett 2011; 307: 37-46. https://doi.org/10.1016/j.canlet.2011.03.013

[2] HAAPANIEMI A, VAISANEN J, ATULA T, ALHO OP, MAKITIE A et al. Predictive factors and treatment outcome of laryngeal carcinoma recurrence. Head Neck 2017; 39: 555-563. https://doi.org/10.1002/hed.24642

[3] KHOUEIR N, MATAR N, FARAH C, FRANCIS E, TAB$\mathrm{CHY} B$ et al. Survival of T4aN0 and T3N+ laryngeal cancer patients: a retrospective institutional study and systematic review. Am J Otolaryngol 2015; 36: 755-762. https://doi. org/10.1016/j.amjoto.2015.07.009

[4] VALERI N, BRACONI C, GASPARINI P, MURGIA C, LAMPIS A et al. MicroRNA-135b promotes cancer progression by acting as a downstream effector of oncogenic pathways in colon cancer. Cancer Cell 2014; 25: 469-483. https:// doi.org/10.1016/j.ccr.2014.03.006

[5] BARTEL DP. MicroRNAs: genomics, biogenesis, mechanism, and function. Cell 2004; 116: 281-297.

[6] VENKATADRI R, MUNI T, IYER AK, YAKISICH JS, AZAD $\mathrm{N}$. Role of apoptosis-related miRNAs in resveratrol-induced breast cancer cell death. Cell Death Dis 2016; 7: e2104. https://doi.org/10.1038/cddis.2016.6

[7] PENG F, ZHANG Y, WANG R, ZHOU W, ZHAO Z et al. Identification of differentially expressed miRNAs in individual breast cancer patient and application in personalized medicine. Oncogenesis 2016; 5: e194. https://doi. org/10.1038/oncsis.2016.4

[8] CALIN GA, CROCE CM. MicroRNA signatures in human cancers. Nat Rev Cancer 2006; 6: 857-866. https://doi. org/10.1038/nrc1997

[9] LIU M, WU H, LIUT, LIY, WANG Fet al. Regulation of the cell cycle gene, BTG2, by miR-21 in human laryngeal carcinoma. Cell Res 2009; 19:828-837.https://doi.org/10.1038/cr.2009.72

[10] CAI KM, BAO XL, KONG XH, JINAG W, MAO MR et al. Hsa-miR-34c suppresses growth and invasion of human laryngeal carcinoma cells via targeting c-Met. Int J Mol Med 2010; 25: 565-571.

[11] KODACH LL, JACOBS RJ, HEIJMANS J, VAN NOESEL CJ, LANGERS AM et al. The role of EZH2 and DNA methylation in the silencing of the tumour suppressor RUNX3 in colorectal cancer. Carcinogenesis 2010; 31: 1567-1575. https://doi.org/10.1093/carcin/bgq147

[12] HSU CH, PENG KL, KANG ML, CHEN YR, YANG YC et al. TET1 suppresses cancer invasion by activating the tissue inhibitors of metalloproteinases. Cell Rep 2012; 2: 568-579. https://doi.org/10.1016/j.celrep.2012.08.030
[13] HU X, ZHANG L, MAO SQ, LI Z, CHEN J et al. Tet and TDG mediate DNA demethylation essential for mesenchymal-to-epithelial transition in somatic cell reprogramming. Cell Stem Cell 2014; 14: 512-522. https://doi.org/10.1016/j. stem.2014.01.001

[14] CHUANG KH, WHITNEY-MILLER CL, CHU CY, ZHOU Z, DOKUS MK et al. MicroRNA-494 is a master epigenetic regulator of multiple invasion-suppressor microRNAs by targeting ten eleven translocation 1 in invasive human hepatocellular carcinoma tumors. Hepatology 2015; 62: 466-480. https://doi.org/10.1002/hep.27816

[15] SUN M, SONG CX, HUANG H, FRANKENBERGER CA, SANKARASHARMA D et al. HMGA2/TET1/HOXA9 signaling pathway regulates breast cancer growth and metastasis. Proc Natl Acad Sci U S A 2013; 110: 9920-9925. https:// doi.org/10.1073/pnas.1305172110

[16] SONG IH. [Cancer metastasis and metastasis suppressors]. Korean J Gastroenterol 2004; 43: 1-7.

[17] HATFIELD S, RUOHOLA-BAKER H. microRNA and stem cell function. Cell Tissue Res 2008; 331: 57-66. https://doi. org/10.1007/s00441-007-0530-3

[18] ZHU S, SI ML, WU H, MO YY. MicroRNA-21 targets the tumor suppressor gene tropomyosin 1 (TPM1). J Biol Chem 2007; 282: 14328-14336. https://doi.org/10.1074/jbc. M611393200

[19] AMBROS V. The functions of animal microRNAs. Nature 2004; 431: 350-355. https://doi.org/10.1038/nature02871

[20] JIN SG, JIANG Y, QIU R, RAUCH TA, WANG Y et al. 5-Hydroxymethylcytosine is strongly depleted in human cancers but its levels do not correlate with IDH1 mutations. Cancer Res 2011; 71: 7360-7365. https://doi.org/10.1158/0008-5472. CAN-11-2023

[21] ZHANG W, LU Z, GAO Y, YE L, SONG T et al. MiR-520b suppresses proliferation of hepatoma cells through targeting ten-eleven translocation 1 (TET1) mRNA. Biochem Biophys Res Commun 2015; 460: 793-798. https://doi.org/10.1016/j. bbrc.2015.03.108

[22] LIN LL, WANG W, HU Z, WANG LW, CHANG J et al. Negative feedback of miR-29 family TET1 involves in hepatocellular cancer. Med Oncol 2014; 31: 291. https://doi.org/10.1007/ s12032-014-0291-2

[23] AHSAN S, RAABE EH, HAFFNER MC, VAGHASIA A, WARREN $\mathrm{KE}$ et al. Increased 5-hydroxymethylcytosine and decreased 5-methylcytosine are indicators of global epigenetic dysregulation in diffuse intrinsic pontine glioma. Acta Neuropathol Commun 2014; 2: 59. https://doi. org/10.1186/2051-5960-2-59

[24] NAVARRO A, YIN P, ONO M, MONSIVAIS D, MORAVEK $\mathrm{MB}$ et al. 5-Hydroxymethylcytosine promotes proliferation of human uterine leiomyoma: a biological link to a new epigenetic modification in benign tumors. J Clin Endocrinol Metab 2014; 99: E2437-2445. https://doi.org/10.1210/ jc. 2014-2264 